\title{
What it Takes for a Successful Mix-and-Inject Serial Crystallography Experiment: From a Sample Preparation and Sample Delivery Perspective Kara Zielinski', Lois Pollack ${ }^{2}$ \\ ${ }^{1}$ Cornell University ${ }^{2}$ Cornell University kaz42@cornell.edu
}

Serial Femtosecond Crystallography (SFX) at X-ray Free Electron Lasers (XFELs) is used to determine room temperature, damage-free, protein structures from micron-sized crystals (Chapman et al. 2011). With its demonstrated success, attention has now turned to Mix-and-Inject Serial Crystallography (MISC), which exploits the small dimensions of the microcrystals to enable rapid mixing for diffusion initiated reactions. Based on our experience with several MISC experiments on different biological systems, we present guidelines for sample preparation and injection to optimize the success of these information-rich experiments.

Samples for MISC must be comprised of a high density $\left(\sim 10^{\wedge} 12\right.$ crystals $\left./ \mathrm{mL}\right)$ of well-diffracting microcrystals. High-throughput screens are a good starting point for identifying promising microcrystallization conditions and crystal seeding is becoming increasing popular for crystal growth optimization. In terms of size, the crystals need at least one dimension below 5 micron to facilitate rapid diffusion, and they should not exceed 10-15 micron in their largest dimension to avoid injector clogging. Other important considerations include protein packing within the crystal, the accessibility of the active site, as well as the size of solvent channels that allow for the transport of ligands into the crystal. Ideally, the sample should be pre-screened to assess ligand binding by an independent technique, such as Electron Paramagnetic Resonance (EPR) spectroscopy (Calvey et al. 2020) or any other appropriately sensitive method.

For sample delivery, Gas Dynamic Virtual Nozzles (GDVNs) are a common choice as they can rapidly introduce fresh crystals for each XFEL pulse. GDVNs produce high-speed jets by exploiting a helium sheath to thin the sample stream down before the liquid exits the nozzle aperture. A robust nozzle design that uses triaxial capillaries can successfully couple a hydrodynamic focusing mixer to a GDVN for MISC (Olmos et al. 2018; Calvey et al. 2016; Calvey et al. 2019). Our lab has developed precise protocols for fabricating versatile mixers with wide sample channels $(50-100 \mu \mathrm{m})$ to reduce clogging, that use relatively low sample flow rates $(3-12 \mu \mathrm{L} / \mathrm{min})$, and have the flexibility to access timepoints ranging from $3 \mathrm{~ms}-2000 \mathrm{~ms}$. These injectors are compatible with different XFEL facilities, and even support vigorous remote operations during the Covid-19 pandemic (Olmos et al. 2018; Pandey et al. 2020).

All of the above, especially the ease of use of mixing injectors in remote operations, demonstrate that MISC is now routine from a sample injection perspective. The current bottleneck for MISC is the creation of high-quality microcrystals in appropriate quantities for XFEL experiments, as well as the need for new techniques to properly characterize samples in advance of beamtimes. 